\title{
Electronic Transport through the Double-Dot System
}

\author{
R. ŚwIRKOWICZ AND D. SzTENKIEL \\ Faculty of Physics, Warsaw University of Technology \\ Koszykowa 75, 00-662 Warsaw, Poland
}

\begin{abstract}
Electron tunnelling through two quantum dots in series is theoretically studied. A limit of intermediate coupling between the dots is considered. The non-equilibrium Green function formalism is used to calculate electric current and mean number of electrons accumulated on the dots. Lesser and retarded Green functions are calculated in the Hartree-Fock approximation with the use of the equation of motion method. Current flowing through the system calculated in dependency on gate voltages shows two resonant peaks, each peak with two additional shoulders. $I-V$ characteristics and differential conductance in a resonance and out of resonance cases are calculated and discussed.

PACS numbers: 75.20.Hr, 72.15.Qm, 72.25.-b, 73.23.Hk
\end{abstract}

\section{Introduction}

Electron transport through a double dot system has recently received much experimental and theoretical attention, especially in Coulomb blockaded and Kondo regimes [1-5]. An important motivation for such investigations is the idea of using these systems for quantum computations [6].

In this work we study transport through double quantum dot (DQD) under Coulomb blockaded conditions. A limit of intermediate coupling between the dots is considered. If the interdot tunnelling is stronger than tunnelling to external reservoirs Hamiltonian of the DQD system can be diagonalized. The double dot can be then represented by an effective single dot with effective tunnelling couplings to external electrodes. In a general case these couplings strongly depend on the interdot coupling. Such an approach to the DQD problem was used by several authors (see e.g. $[3,7]$ ). Those calculations were based mainly on a master equation. Linear conductance as a function of a gate voltage was mainly investigated $[3,7,8]$. Here, we use the non-equilibrium Green function formalism to study electron transport through the system. The approach allows us to find $I-V$ characteristics, differential conductance as well as mean number of electrons accumulated on the dots. 


\section{The model}

Hamiltonian of the system consisting of two quantum dots in series attached to external reservoirs is taken in the form

$$
H=H_{L}+H_{R}+H_{2 \mathrm{D}}+H_{T} .
$$

Terms $H_{\beta}=\sum_{k \beta} \varepsilon_{k \beta} a_{k \beta}^{+} a_{k \beta}$ with $\beta=L, R$ describe left $(\beta=L)$ and right $(\beta=R)$ electrodes in the non-interacting particle approximation. $H_{2 \mathrm{D}}$ describes DQD and is equal to

$$
H_{2 \mathrm{D}}=E_{L} d_{L}^{+} d_{L}+E_{R} d_{R}^{+} d_{R}+U n_{L} n_{R}+W\left(d_{L}^{+} d_{R}+\text { h.c. }\right) .
$$

Only one energy level $E_{\beta}$ on the $\operatorname{dot} \beta$ is assumed to be active in the transport. Experiments are mainly performed in such conditions that Coulomb charging energy and excitation energy are much larger than $k T$. Accordingly, only a single state on each dot is important and double occupancy of each dot can be ignored. So, we consider only empty or singly occupied states and omit in Hamiltonian (2) a term describing intradot Coulomb interaction. The interdot Coulomb interaction is taken into account via the Hubbard term with the correlation parameter $U$. The last term in Hamiltonian $H_{2 \mathrm{D}}$ (Eq. (2)) describes tunnel interdot coupling with the strength $W$. Tunnelling processes between DQD and the external electrodes are included in $H_{T}$ (Eq. (1)) which takes a form: $H_{T}=\sum_{k \beta}\left(t_{k \beta} a_{k \beta}^{+} d_{\beta}+\right.$ h.c.), $t_{k \beta}$ denotes here a tunnelling amplitude.

The Hamiltonian $H_{2}$ D can be diagonalized by means of a unitary transformation [7]. The transformation allows us to represent DQD by a two-level effective dot. The procedure is justified when the interdot tunelling is stronger than couplings to external electrodes. This new system can be described by the following Hamiltonian:

$$
H=H_{L}+H_{R}+\sum_{i=1}^{2} E_{i} c_{i}^{+} c_{i}+U c_{1}^{+} c_{1} c_{2}^{+} c_{2}+\sum_{k \beta i}\left(T_{k \beta}^{i} a_{k \beta}^{+} c_{i}+\text { h.c. }\right),
$$

$E_{i}$ are energy levels of the effective dot. They depend on $E_{L}, E_{R}$, and the interdot coupling $W[7]$. Matrix elements $T_{k \beta}^{i}$ describe tunnelling processes between the two-level dot and the electrodes [7].

\section{Non-equilibrium Green function formalism}

To study electron transport through the system described by the Hamiltonian (3) we introduce the retarded $G_{i j}^{r}$ (advanced $G_{i j}^{a}$ ) and lesser $G_{i j}^{<}$Green functions, which are defined in a standard way [9]. The equation of motion method is used to calculate both retarded and lesser functions in out of equilibrium state [10]. Higher order functions are split according to Hartree-Fock approximation. The same approximation scheme is used for $G^{r}$ and $G^{<}$. So, both functions are calculated on equal footing. 
Electric current $I$ flowing through the system with a bias voltage applied can be expressed in the form [11]

$$
\begin{aligned}
I= & \frac{e}{2 \hbar} \int \frac{\mathrm{d} E}{2 \pi} \sum_{i}\left[\tilde{\Gamma}_{i i}^{L} \Gamma_{i i}^{R}+\tilde{\Gamma}_{i i}^{R} \Gamma_{i i}^{L}+\frac{1}{2}\left(\tilde{\widetilde{\Gamma}}_{i-i}^{L} \Gamma_{i-i}^{R}+\tilde{\widetilde{\Gamma}}_{i-i}^{R} \Gamma_{i-i}^{L}\right)\right] \\
& \times\left|G_{i i}\right|^{2}\left(f_{L}-f_{R}\right)
\end{aligned}
$$

and a mean number of electrons on the level $i$ is calculated according to the formula

$$
n_{i}=\left\langle c_{i}^{+} c_{i}\right\rangle=i \int \frac{\mathrm{d} E}{2 \pi} G_{i i}^{<}=\int \frac{\mathrm{d} E}{2 \pi}\left(\tilde{\Gamma}_{i i}^{L} f_{L}+\tilde{\Gamma}_{i i}^{R} f_{R}\right)\left|G_{i i}\right|^{2} .
$$

In the above expressions $f_{\beta}$ denotes the Fermi-Dirac distribution function. $\Gamma_{i j}^{\beta}$ can be expressed in terms of the bare couplings $\Gamma^{\beta}=2 \pi \sum\left|t_{k \beta}\right|^{2} \delta\left(\varepsilon-\varepsilon_{k \beta}\right)$, the interdot coupling $W, E_{L}$, and $E_{R}[7] . \tilde{\Gamma}_{i i}^{\beta}$ and $\widetilde{\widetilde{\Gamma}}_{i-i}^{\beta}$ describe here certain effective coupling parameters which are related to $\Gamma_{i j}^{\beta}[11]$. One can see (Eq. (5)) that $\tilde{\Gamma}_{i i}^{\beta}$ determine $G_{i i}^{<}$function. Similarly, $\widetilde{\widetilde{\Gamma}}_{i-i}^{\beta}$ determine $G_{i-i}^{<}$. Electric current $I$ and $n_{i}$ are calculated self-consistently.

\section{Numerical results}

Formalism described in the previous section allows one to investigate a general case with DQD coupled symmetrically or asymmetrically to the external leads. Here, we discuss and present numerical results only for the symmetrical case. Tunnelling couplings $\Gamma^{L}, \Gamma^{R}$ between DQD and the two electrodes are assumed to be the same and equal to $\Gamma$. In the following $\Gamma$ is treated as an energy unit. Calculations are performed for interdot correlation parameter $U=16 \Gamma$ and temperature $T=\Gamma / k$. The interdot coupling $W$ is treated as a tunable parameter. Positions of energy levels $E_{L}$ and $E_{R}$ in the coupled dots can be independently changed by gate voltages $V_{g L}, V_{g R}$ applied to $L$ and $R$ dots, respectively.

Figure 1 presents in the grey scale electric current $I$ flowing through the DQD system under a bias voltage $V=2 \Gamma /|e|$ in dependence on gate voltages $V_{g L}, V_{g R}$. The figure shows that transport is possible only for certain values of gate voltages. There are two sharp resonance peaks when energy levels of the effective dot $E_{i}$ or $E_{i}+U$ enter a tunnelling window. When gate voltages $V_{g L}$ or $V_{g R}$ are swept electrochemical potentials of the $L$ and $R$ dots can be aligned and resonant tunnelling can take place. The current is enhanced along the solid line, for which this condition is fulfilled. However, each peak shows two additional shoulders marked in the figure with dashed lines. The shoulders appear when the level $E_{1}$ or $E_{2}$ of the effective dot enters a tunnelling window. Some changes in $E_{L}$ or $E_{R}$ induced by gate voltages $V_{g L}$ or $V_{g R}$ do not change substantially $E_{1}\left(E_{2}\right)$, so the levels still appear in the tunnelling window. Increasing $W$, i.e. the strength of the interdot coupling, produces more pronounced shoulders. The results are consistent with the ones calculated by Ziegler et al. with the use of a master equation [7]. 


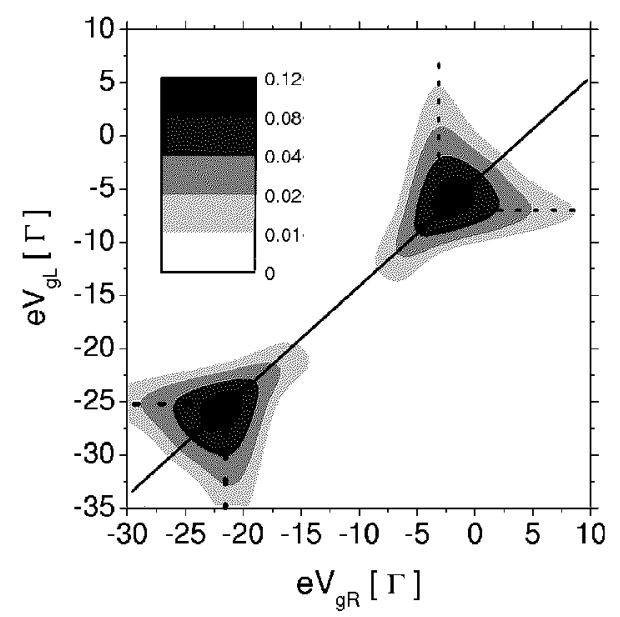

Fig. 1. Electric current in a grey scale in dependence on gate voltages calculated for $W=2 \Gamma, E_{L}=4 \Gamma, V=2 \Gamma /|e|$.

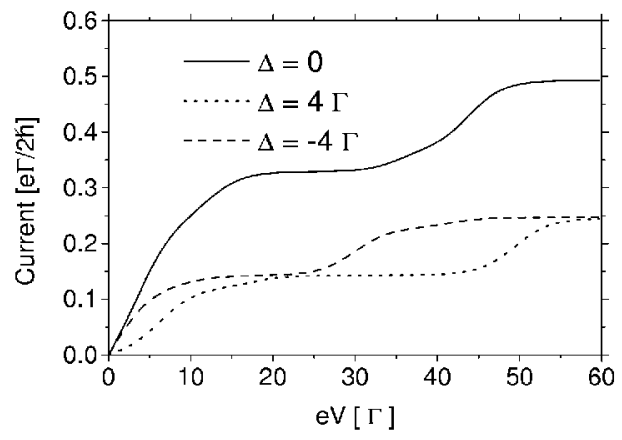

Fig. 2. Current in dependency on bias voltage for $W=2 \Gamma$ in a resonant case $\Delta=0$ and out of resonance cases $\Delta=4 \Gamma, \Delta=-4 \Gamma$.

$I-V$ characteristics are presented in Fig. 2 for interdot coupling $W=2 \Gamma$. Characteristics are taken for three different situations, namely in the resonant case with $\Delta=E_{R}-E_{L}=0$ and in two out of resonance situations with $\Delta=-4 \Gamma$ and $\Delta=4 \Gamma$. The current through the DQD system is resonantly enhanced when the levels of $L$ and $R$ dots are aligned, i.e. $E_{L}=E_{R}$. In out of resonance cases $(\Delta=-4 \Gamma, \Delta=4 \Gamma)$ current is much smaller. In all cases the current shows a stepwise increase with increasing bias voltage. The step appears when an additional level enters a bias window, so an extra transport channel opens. Figure 2 shows that only some current steps are well visible. To analyze the problem we calculate the differential conductance $G_{\text {diff }}$.

In the resonant case $\left(\Delta=0, E_{L}=E_{R}\right) G_{\text {diff }}$ shows four well-defined peaks (Fig. 3a). A peak in $G_{\text {diff }}$ appears when a mean number of electrons $n_{i}=\left\langle c_{i}^{+} c_{i}\right\rangle$ $(i=1,2)$ accumulated on level $E_{i}$ of the effective dot increases. It can be seen in 

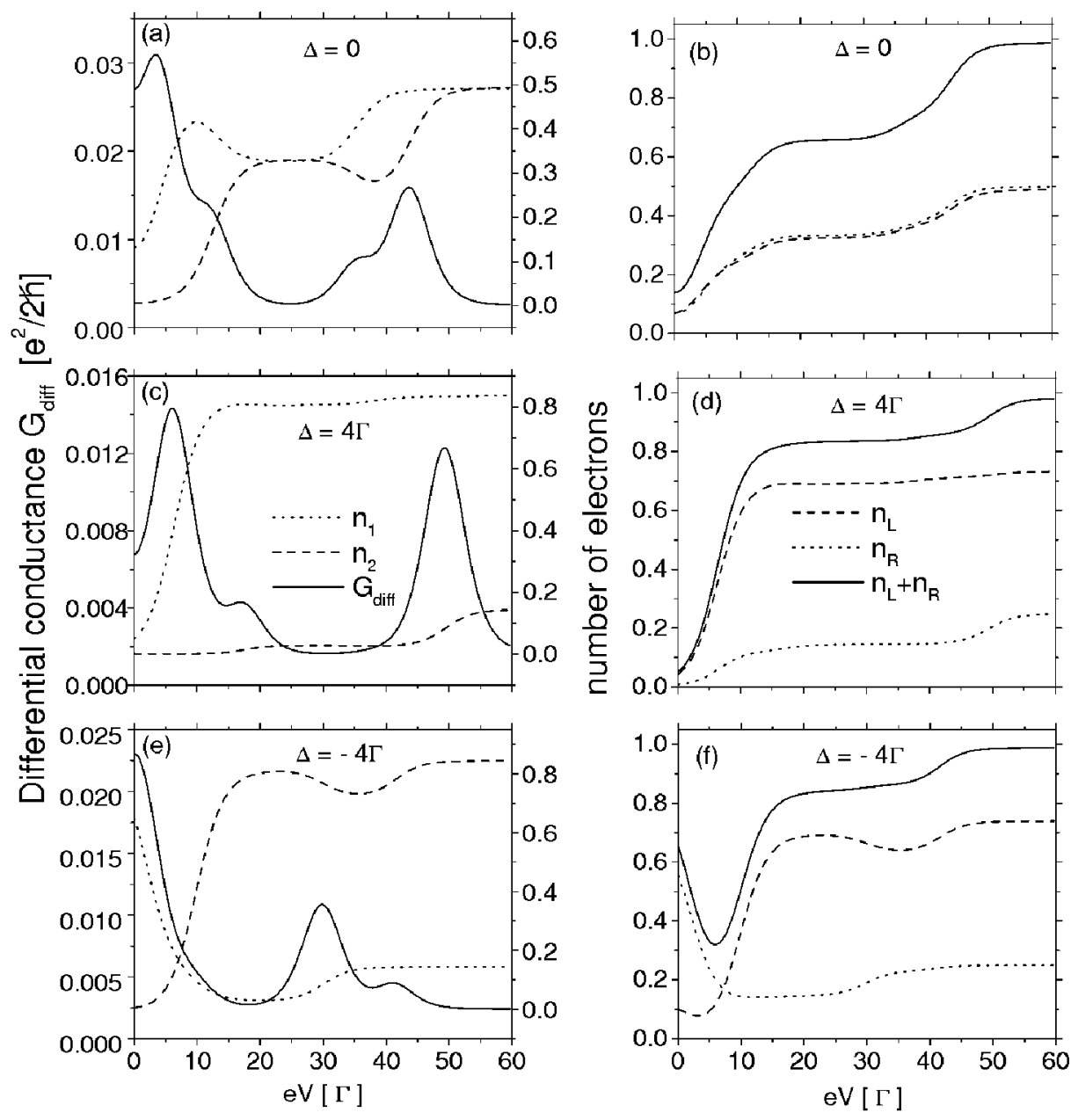

Fig. 3. Left panel: Differential conductance (solid line) and mean numbers of electrons $n_{1}$ (dotted line) and $n_{2}$ (dashed line) as functions of bias voltage for $W=2 \Gamma$ in a resonant case $\Delta=0$ (a) and out of resonance cases $\Delta=4 \Gamma$ (c), $\Delta=-4 \Gamma$ (e). Right panel: Number of electrons $n_{L}, n_{R}$ on left and right dots.

the figure where $n_{1}$ and $n_{2}$ are also presented as functions of bias voltage. In the region of small voltages the low-energy level $E_{1}$ is mainly occupied. An increase in $n_{2}$ leads to a decrease in $n_{1}$ which is a result of Coulomb repulsion $U$ between electrons. In the resonant case with energy levels of the both dots aligned a mean number of electrons $n_{\beta}=\left\langle d_{\beta}^{+} d_{3}\right\rangle(\beta=L, R)$ localized on the left and right dot is practically the same (Fig. $3 \mathrm{~b}) . n_{\beta}$ shows a stepwise increase with bias voltage increasing.

In out of resonance case with $\Delta=4 \Gamma$ three peaks in $G_{\text {diff }}$ are visible (Fig. $3 \mathrm{c}$ ). In this case the distance between levels $E_{1}$ and $E_{2}$ of the effective dot is quite con- 
siderable. The energy level $E_{1}$ starts to fill up and becomes almost fully occupied at relatively low voltages, whereas $E_{2}$ remains empty (Fig. 3c). A strong increase in $n_{1}$ leads to a high peak in the conductance. An increase in $n_{2}$ gives only a cusp in $G_{\text {diff. }}$. In the high voltage region $n_{1}$ is practically constant but $n_{2}$ starts to increase when $E_{2}+U$ enters a tunnelling window. So, for high voltages only one peak corresponding to $E_{2}+U$ can be observed in $G_{\text {diff }}$.

For $\Delta=-4 \Gamma$ the main peak in $G_{\text {diff }}$ corresponds to the zero bias and no blockade is obtained (Fig. 3e). In such a situation the right dot is almost half-occupied, the left one is almost empty and the current can flow through the system under a small bias voltage applied. With increasing $V$ a mean number of electrons on the dot $R-n_{R}$ diminishes substantially and a number of electrons on the left one $-n_{L}$ starts to increase (Fig. 3f). Similarly, $n_{1}$ decreases and the level $E_{1}$ becomes almost empty, whereas $n_{2}$ increases and the level $E_{2}$ starts to fill up (Fig. 3e). The peak which corresponds to an increase in $n_{2}$ overlaps with the main peak in $G_{\text {diff }}$, so the two peaks are not resolved. Only some enhancement in $G_{\text {diff }}$ can be observed. In the high voltage regime occupation numbers $n_{1}$ and $n_{2}$ start to increase when levels $E_{1}+U$ and $E_{2}+U$ one after the other enter a tunnelling window. The appropriate peaks are well visible in $G_{\text {diff }}$. However, amplitudes of the peaks are different.

Close relations between occupation numbers of the two coupled dots and positions as well as amplitudes of the conductance peaks for a resonant case and out of resonance ones were found by Klimeck et al. [8]. However, they discussed a linear conductance $G$, whereas we present results obtained for the differential one $G_{\text {diff }}$.

\section{References}

[1] W.G. Van der Wiel, Rev. Mod. Phys. 75, 1 (2003).

[2] C.A. Stafford, R. Kotlyar, S. Das Sarma, Phys. Rev. B 58, 7091 (1998).

[3] K.A. Matveev, L.I. Glazman, H.U. Baranger, Phys. Rev. B 54, 5637 (1996).

[4] C. Niu, L. Liu, Phys. Rev. B 51, 5130 (1995).

[5] T. Pahjola, J. Konig, M.M. Salomaa, J. Schmid, H. Schoeller, G. Schon, Europhys. Lett. 40, 180 (1997); R. Aguado, D.C. Langreth, Phys. Rev. B 67, 245307 (2003); Qing-feng Sun, H. Guo, Phys. Rev. B 66, 155308 (2002).

[6] D. Loss, D.P. DiVincenzo, Phys. Rev. B 57, 120 (1998).

[7] R. Ziegler, C. Bruder, H. Schoeller, Phys. Rev. B 62, 1961 (2000).

[8] G. Klimeck, G. Chen, S. Datta, Phys. Rev. B 50, 2316 (1994).

[9] H. Haug, A.P. Jauho, Quantum Kinetics in Transport and Optics of Semiconductors, Springer Verlag, Berlin 1996.

[10] C. Niu, D.L. Lin, T.H. Lin, J. Phys, Condens. Matter 11, 1511 (1999).

[11] R. Świrkowicz, J. Barnaś, M. Wilczyński, to be published. 